\title{
Aproximación al estado del arte de procesos de aprendizaje en educación virtual
}

Dignora Inés Páez Giraldo ${ }^{1}$ María Eugenia Navas Ríos ${ }^{2}$

\section{Resumen}

El artículo presenta una aproximación a lo que se ha investigado acerca de los procesos de aprendizaje en Educación virtual, realizando un barrido en medios electrónicos e impresos a nivel nacional einternacional en los últimos diez años, debido al creciente interés de la comunidad académica en este tema.

La producción cientifica sistematizada, que ha hecho aportes relevantes en este ámbito educativo, se presenta organizada inicialmente desde las fuentes (artículos, tesis, proyectos de investigacion, memorias de congresos); a șu vez, desde cada fuente se hace una clasificación de los temas tratados, según categorías como pedagogía, didáctica, calidad de la educación virtual y éica y valores, las cuales emergen del mismo análisis. Por último, se presenta una aproximación a la tendencia por países en cuanto al interés por investigar la educación abierta y a distancia.

Los hallazgos dentro de cada grupo introducen elementos significativos que apuntan a la transformación del quehacer del docente y del discente, posibilitándose la pertinencia y calidad en la oferta de estos programas académicos en todos los niveles educativos, revaluando prejuicios que al respecto se tienen sobre este tipo de mediacion virtual.

La investigación es de tịpo descriptivo y se ha utilizado como método de investigacion el analisis documental. La seleccion de los documentos

\footnotetext{
1 Licenciada en filosofía, Magister en educación, estudiante de Doctorado en Ciencias de la Educación. Docente de la Escuela de Ciencias de la Educación, Universidad Nacional Abierta y a Distancia, UNAD. Directora del Grupo Giuc. Email: dignora.paez@unad.edu.co.Colombia.

2 Magister en Educación, Doctora en Ciencias de la Educación. Docente Universidad de Cartagena. Email: mariaeunavas@ yahoo.com. Colombia.
} 


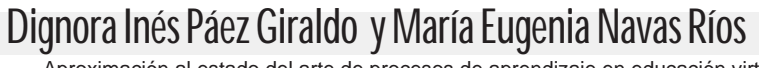

Aproximación al estado del arte de procesos de aprendizaje en educación virtual, artículo producto de la investigación

impresos y online seha realizado por medio de un muestreo deconveniencia de los investigadores, teniendo como único criterio de selección la pertinencia de los conocimientos con el objeto del trabajo.

Balabbras clawe: Educación, virtualidad, pedagogía, didáctica, autonomía, ética.

\section{An approach to the state of the art in virtual education learning processes}

\begin{abstract}
A.bstract
This paper approaches the corpus of research on learning processes in virtual education by examining electronic and printed media in our country and abroad. This has been done in the last 10 years due to the growing interest in these issues among the academic community.

Scientific production, to which system analysts have done relevant contributions, appears initially organized from the sources (articles, thesis, investigation projects, conference reports). In turn, from each source, the various points of view are classified in the following categories: pedagogy, didactics, quality of the virtual, and ethical education and values. These categories arise from the same analysis. Finally an approach to current trends within various for countries is presented to address the interest in investigating the Distance Education system. Findings within every group introduce significant elements pointing out to the transformation of teacher and student roles in the education sector. It shows the relevancy and quality these academic programs offer in all educational levels, reviewing prejudices about this matter of virtual education.
\end{abstract}

The research is of the descriptive type and the method of investigating the documentary analysis was used. The selection of printed and online documents result ted from a sampling that suited the researchers' purpose under the criteria of the relevancy of knowledge regarding the topic.

Key words: Education, virtuality, pedagogy, didactics, autonomy, ethics. 
Revista de

investigaciones UNAD

Volumen 10. Número 1. Junio 2011

\section{Introducción}

Según reflexiones, de la U niversidad Oberta de Catalunya (2004), "Ia relación entre las, Tecnologías de la Información y la Comunicacion (TIC) y la mejora de las practicas educativas, confirman como esta dista de ser lineal o sencilla. Las TIC abren, sin duda, por sus propias caracteristicas, nuevas posibilidades de innovacion y mejora de los procesos formales de enseñanza y aprendizaje, pero la mera incorporación de herramientas tecnologicas a las practicas educativas no garantiza en modo al guno que esa mejora se produzca realmente". Estas herramientas se convierten en retos para las instituciones de Educacion Superior, y de manera especial para las Instituciones de Educacion a Distancia con mediaciones virtuales.

En la última década la incursión de las TIC en el campo educativo ha sido enorme; por ello se considero pertinente realizar una aproximacion al estado del, arte de los procesos de aprendizaje en Educacion virtual, valiéndose de articulos publicados, proyectos de investigacion, tesis y memorias de congresos que permitieran mostrar el estado actual desde una investigacion descriptiva y abordar lo que se ha investigado y socializado en el mundo y en Colombia, en relacion con la educación virtual.

Los hallazgos sistematizados se presentan siguiendo la metodología de Sandoval (1999), desde la recoleccion hasta el análisis de los documentos y la presentacion de los resultados encontrados, que guardan el sigulente orden: en primera instancia se presenta sistematizada la produccion cientifíca por fuentes y categorias: pedagogia didactica, calidad de la educacion virtual y etica y valores. L uego, en igual forma, se presenta la produccion cientifica hallada, pero desde los paises de origen, lo que lleva a concluir desde la produccion, cuál es, la tendençia en la uiltima decada en cuanto al grado de interes por categoría y por pais.

Es prioritario mantener y avivar la reflexión sobre la Educación Superior Virtual, los objetivos y criterios que deben guiar la incorporacion de las TIC a las practicas educativas y la importancia de la investigacion empirica sobre los resultados y aportaciones de dicha incorporacion para la mejora de la calidad de la enseñanza.

\section{Metodología}

La investigación es de tipo descriptivo y la metodolo lóía empleada en el desarrollo de la investigación fue el análisis e interpretacion de documentos. De ahi que el proceso de desarrollo de la investigación se dividio siquiendo cada una de las cinco fases que implica esta metodología, en opinion de Sandoval (1999). 


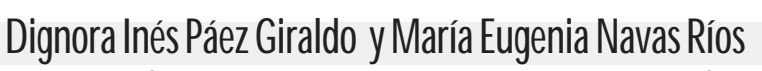

En la primera etapa se realizael rastreo einventario de los documentos existentes y disponibles. Para este trabajo, la selección de los documentos impresos y online se hizo por medio de un muestreo de conveniencia de los investigadores, teniendo como unico criterio de seleccion la pertinencia de los conocimientos con el objeto del trabajo; en la segunda etapa hay que clasificar los documentos identificados; en la tercera es necesaria la seleccion de los documentos ya clasificados, de manera que sean los mas pertinentes para los propositos dela investigacion; en la cuarta la importancia radica en la lectura a protundidad del contenido de los documentos seleccionados, para extraer elementos de análisis y consignarlos en archivos de Word atendiendo a los patrones, tendencias, convergencias y contradicciones que se vayan descubriendo; por ultimo, en la quinta etapa es fundamental hacer una lectura cruzada y comparativa de los documentos en cuestion, no sobre la totalidad del contenido de cada uno sino sobre los hall azgos previamente hechos, de modo que sea posible construir una sintesis comprensiva sobre la realidad de lo virtual.

Las categorías que surgieron frente a la clasificación de los trabajos investigativos fueron:

Pedagogía: Entendida como un discurso metódico capaz de articular una forma de ser del maestro, unos conocimientos claros, un lugar determinado (la escuela), unos contenidos y una caracterización de la forma de aprender.

Didáctica: vista como la ciencia y el arte de enseñar; es ciencia en cuanto investiga y experimenta nuevas tecnicas de enseñanza y es arte porque establece normas de accion o sugiere normas de comportamiento didactico, basándose en los datos científicos y empiricos de la educacion.

Calidad de la educación virtual: proceso de enseñanza-aprendizaje virtual, interiorizado como una forma de hacer las cosas en la que fundamental mente predomina la preocupacion por satisfacer al que aprende y por mejorar, dia a dia, procesos y resultados. El concepto actual de calidad ha evolucionado hasta convertirse en una forma de gestion que introduce el concepto de mejora continua en cualquier organización y a todos los niveles de la misma, y que afecta a todas las personas y a todos los procesos.

Ética y Valores: concebidos como una disciplina filosófica que reflexiona sobre el "ethos" cultural y aborda valores como la autonomía.

\section{Resultados}

En el desarrollo de la primera etapa se evidenció que el mayor número de publicaciones corresponde a articulos sobre el tema de lo virtual; luego 
Revista de

investigaciones UNAD

Volumen 10. Número 1. Junio 2011

aparecen las memorias de congresos y al gunas publicaciones de proyectos de investigacion totalmente concluidos, y finalmente, las tesis.

En la segunda etapa se clasificó la producción sobre lo virtual desde cuatro categorias: pedagogía, didactica, calidad de la educación y ética y valores, surgidas del mismo análisis de la documentación seleccionada. Esta etapa se efectuo en forma paralela con la tercera, en la medida en que se agrupaban las publicaciones y se iban seleccionando las de mayor aporte y pertinencia al tema abordado desde la investigacion.

Finalizadas la cuarta y quinta etapas de la metodología del análisis de documentos, se encontraron los siguientes resultados:

1. Resultados de la producción cientifica por fuentes y categorias: pedagogía, didáctica, calidad de la educación virtual y ética y valores.

a) En los artículos publicados existe una gran preocupación, en lo virtual, por estilos, modos y medios de aprendizaje, las transformaciones del aula, el rol cambiante del docente y las aportaciones de las tecnologias. Por esta razon, hay una variedad de produccion escrita acerca de la Didáctica en los ambientes virtuales, que ocupa el primer lugar en cuanto al numero de publicaciones, con $53 \%$, según la figura 1.

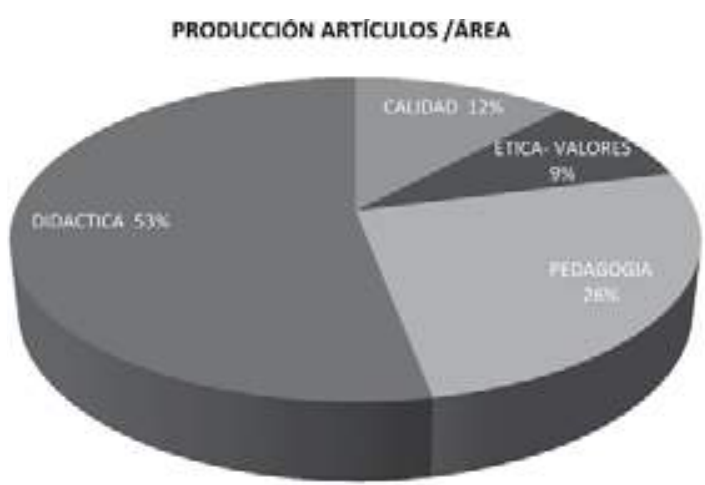

Figura 1. Publicación de artículos por áreas y/o categorías Fuente: Construcción propia de las autoras

Le sigue en su orden la categoría Pedagogía con un 26\%, de participación, que contiene una serie de escritos que tienen como proposito fundamentar la conceptual izacion y contextualizacion del proceso enseñanza-aprendizaje en la virtualidad, mirado como un todo complejo y revolucionario que exige diferentes transformaciones. 


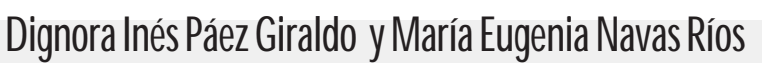

Aproximación al estado del arte de procesos de aprendizaje en educación virtual, artículo producto de la investigación

Uno de los artículos analizados pregunta: iexiste la educación virtual? A este interrogante responden muchos de los articul os encontrados. Se resaltan las respuestas dadas por Gámez y Cabrero. Gamez, (2002), especialista en educacion virtual, responde categóricamente que si existe, que es una concepcion nueva y muy debatida actualmente, y se apoya en dos premisas: 1) la preocupacion por potencializar maneras de solucionar ambientes del proceso enseñanza aprendizaje, y, 2) la virtualidad, que úlimamente se vincula con las instituciones de educacion superior.

Vale la pena reflexionar sobre las posibilidades de desempeño en una actividad social y profesional. Cabrero (2005), por su parte, asegura que en un entorno virtual se puede crear "una verdadera comunidad de personas" y conseguir "relaciones mas cercanas" que en un sistema presencial, apoyando desde este punto de vista la existencia de la educacion virtual.

Dentro de esta categoría se incluyó, además, toda la producción científica relacionada con el nacimiento y desarrollo de de la enseñanza y aprendizaje abierto y a distancia y las tendencias de la virtual idad. A manera de ejemplo se trae el articulo "Historia de la Educación a D istancia", escrito por Garcia A retio (2005). Resumiendo, demuestra cómo el nacimiento y posterior desarrollo de la enseñanza y aprendizaje abierto y a distancia se ha producido desde "los avances sociopolíticos, la necesidad de aprender a lo largo de la vida, la carestía de los sistemas convencionales, los avances en las Ciencias de la Educacion y las transformaciones tecnologicas". La evolucion de la educacion abierta y a distancia la clasifica en tres grandes momentos: el primer momento lo denomina correspondencia; el segundo telecomunicación y el tercero, que corresponde a lo actual, el momento que vivimos, telematica.

Las categorías Calidad de la Educación virtual (12\%) y Ética y valores (9\%) son las de menor produccion. L a primera contiene autores que en sus escritos se definen como dedicados a refl exionar sobre al gunos componentes de la cal idad de la Educacion virtual; en esta categoria hay, ademas, produccion desde el Estado por intermedio de sus organismos representativos.

En cuanto a la concepción de la calidad de la educación virtual, mencionamos a B eatriz Fainholc quien manifiesta que se trata de un sistema muy complejo opinion que comparten las autoras de estetrabajo, porque además de la densidad del proceso de enseñanza-aprendizaje visto desde las personas que intervienen en el, la diversidad de los métodos, metodologias y procesos y el contexto socjocultural, se carece de experiencia y tradicion en el manejo de las TIC, que estan en permanente evolucion, lo cual nos condena a ser interminablemente inmigrantes digitales.

Teresa M auri y otros, se dedican a anal izar la cal idad de los contenidos educativos reutilizables, especificamente, calidad en el diseño, y usabilidad (entendida como 


\section{Revista de}

una oportunidad que brindan estos tipos de diseños de contenidos virtuales), los cual es pueden ser empl eados de manera intra e interinstitucional.

En cuanto a la producción por parte del Estado, encontramos escritos producidos y publicados en Colombia por entes administrativos como el Instituto Colombiano para el fomento de la Educación Superior, Icfes y el Conseio Nacional de acreditación. CNA. Esta entidad publico en noviembre de 2006 dentro de la serie Documentos Especiales el No. 3 denominado "Indicadores para la autoevaluacion con fines de acreditación de programas de pregrado en las modalidades a distancia y virtual". En él concibe la educación superior abierta y a distancia como una estrategia metodo lógica de organizacion y administracion que orienta una serie de factores con sus respectivas características e indicadores de obligatorio cumplimiento para todos aquellos programas que se ofrecen bajo esta modalidad como le ha llamado el mismo - stado colombiano. El cumplimiento de las directrices, denominadas por el Estado condiciones de calidad, asegura el otorgamiento por parte del Estado del respectivo Registro Calificado para que la Institución proceda a hacer legalmente su ofrecimiento.

Pasando a la categoría Ética y valores, es muy poca la producción científica de los investigadores. Se resaltan los escritos de A dela Ros $(s / f)$ de España quien reflexiona sobre los aspectos sociales del e-learning y del colombiano Guillermo Cardona $(s / f)$, profesor de la Universidad Pedagogica Nacional de Colombia, quien analiza la relacion entre la educacion virtual y las necesidades humanas.

b) En lo relacionado con las tesis e investigaciones, se halló que el $87 \%$ de la producción se encuentra ubicada en el área de la pedagogia y el 13\%, en el área de la didactica (ver figura 2).

\section{Tesis e Investigaciones/ Área}

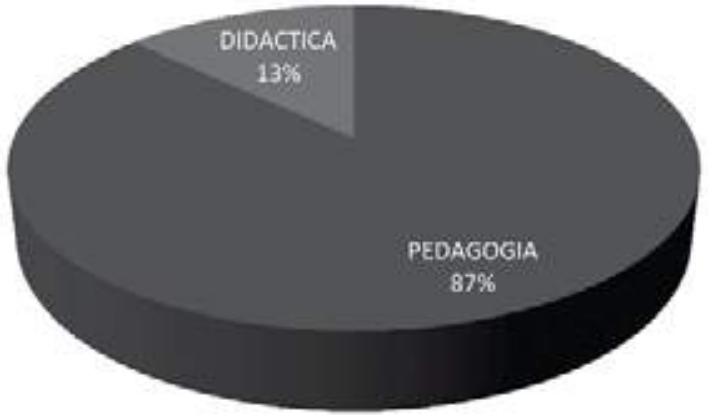

Figura 2. Porcentaje de publicaciones de tesis e investigaciones por área

Fuente: Construcción propia de las autoras 


\section{DigaralrésPázGrddbyMariaEtgeriaNbuesRos}

Aproximación al estado del arte de procesos de aprendizaje en educación virtual, artículo producto de la investigación

Los temas mayormente tratados en la categoría de Pedagogía son los rel acionados conla inauguración de un nuevo model o pedagogico sustentado fundamentalmente en el campus virtual, lo que permite nuevas formas de interaccion social entre los miembros de esta comunidad educativa. Esta propuesta lleva a interrogarse sobre la manera como se ejercen las relaciones de poder en estos espacios y como el individuo se convierte en un codigo, vigilado y controlado por sistemas de ensamblajes tecno ogicos que controlan, su acceso, le imponen una identidad, le exigen participacion y conducen su movimiento.

En el mismo sentido, Vanegas (2002) afirma que los entornos virtual es establecen nuevas formas de ejercicio del poder en las cuales la vigilancia disciplinaria cede su lugar a formas de control abierto y continuo soportado en las nuevas tecnologias informáticas.

Dentro del área de lo didáctico, se trae como ejemplo el trabajo de Cenich y otros (2004). En el trata el tema de la implementacion del aprendizaje a traves de proyectos y trabajo colaborativo, como experiencias de cursos en línea, basados en los beneficios de disenar proyectos autenticos en ambientes virtuales de aprendizaje con un conjunto de herramientas que soportan la comunicacion online para trabajar col aborativamente (página web, grupo de discusion y correo electrónico). Collins (1997) hace su aporte con un trabajo en el que se destaca la construcción de las funciones del tutor a partir de la reflexion, de las actuaciones de un experto (tutor del curso) y del propio desempeño del alumno a distancia.

Por su parte, Camacho (2006) escribe sobre las competencias específicas del docente-tutor en la virtuali dad y proponeformar y cualificar al tutor en el desarro|lo de las competencias de abstraccion, pensamiento critico, experimentacion, investigacion, colaboracion y cooperacion de los estudiantes borrando con esto los limites que se habían establecido desde la génesis de la educación a distancia y posicionando al tutor en la produccion de pensamiento pedagogico.

Dentro de este grupo de producción científicca enmarcada en la categoría didáctica, se pudo percibir las diferentes designaciones que ha recibido la educacion a distancia a lo largo de estos 10 años analizados, tales como: educación no formal, de masas, por correspondencia, informal, virtual, mediada, abierta, entre otras. Esto nos lleva a concluir que en cada etapa este tipo de educacion ha tenido que justificar su existencia; sin embargo, estas concepciones no han sido obstaculo para continuar su presencia educativa desde la ensenanza por correspondencia hasta la multimedia, la telematica y la via internet.

Resumiendo, con relación a la didáctica, los temas relevantes son, entre otros: Estrategias de evaluación con sus respectivos instrumentos, criterios eindicadores adecuados para evaluar las distintas actividades como chats, foros, diarios individuales y entre pares, wikis, ensayos, informes y portafolios. Se resalta que todas estas actividades estan mediadas por las TIC. Al respecto, Bernal (2006) 
Revista de

investigaciones UNAD

Volumen 10. Número 1. Junio 2011

afirma que las $\mathrm{TIC}$ son útil es para generar un cambio conceptual en los estudiantes e implementar unidades didácticas que proporcionen una vision holistica del conocimiento a quienes participan de los procesos educativos.

c) En cuanto a la publicación de las memorias de congresos, naturalmente existe un tema central sobre el cual gira el congreso y es importante la participacion de los asistentes. Estos son los resultados en las categorias en estudio: $56 \%$ de publicaciones dedicadas a reflexionar diverssos temas en la categoria de Didacticas en educación virtual; $33 \%$ en la categoría Pedagogía y un $11 \%$ en la categoría Etica y valores. (Ver figura 3).

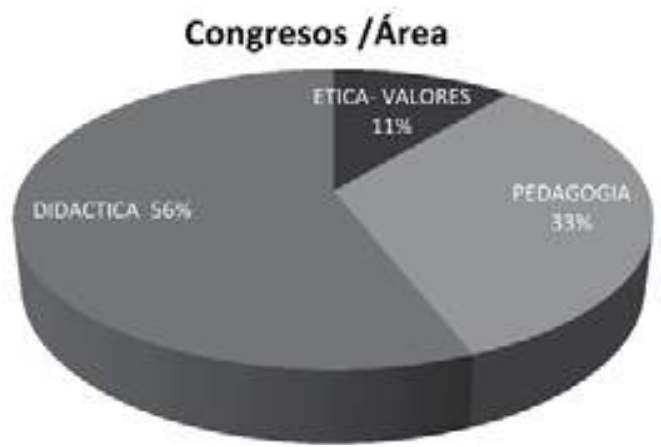

Figura 3. Porcentaje de publicaciones de memorias de congresos por área

Fuente: Construcción propia de las autoras

Los temas en la categoría Pedagogía giraron en torno al interés y la necesidad de construir modelos pedagogicos o educativos para la educacion a distancia.

Los eventos internacionales sobre educación a distancia y virtual presentan un numero, considerado de ofertas. En el III Congreso Online OCS se discute como en A mérica, Latina subsisten brechas no solo en lo economico sino en lo social y lo tecnológico. Desde alli se invita a que las investigaciones en este campo deben desarrollarse paralelamente con proyectos dirigidos a lograr equidad en el acceso a esta clase de educacion y mayores niveles de autonomia y autogestión en los aprendizajes como resultado principal de la intervencion y la mediacion de entornos virtuales. También se plantea que la revolucion del pensamiento implica una verdadera transformación educativa.

Las teorías o corrientes pedagógicas han oscilado entre enfoques regularmente centrados en el docente o en el estudiante. En el mundo de la globalidad, las sociedades se debaten en la tranșicion para llegar a constituirse en sociedades de la informacion, del conocimiento o del aprendizaje, sustentadas en las nuevas tecnologias de la informacion. Ante estos retos, es necesario 


\section{DigoralnésPázGradobyMariaEtgriankavesRós}

Aproximación al estado del arte de procesos de aprendizaje en educación virtual, artículo producto de la investigación

replantear el quehacer pedagógico como base para formar al nuevo ciudadano. Sus escenarios demandan una nueva arquitectura educativa que apunte al aprendizaje en y para la vida, lo que implica promover una nueva hipotesis educativa: Hay que enseñar a aprender a aprender para que el estudiante de manera autónoma desarrolle diversas competencias que le permitan un mejor manejo de la información y el conocimiento.

Siendo consecuentes con lo planteado, se requiere un nuevo enfoque para comprender el quehacer educativo, ante lo cual el docente debe asumir un rol de mediador entre los estudiantes y la informacion existente para que esta sea punto de partida y de llegada en el proceso de enseñanza-aprendizaje. El centro escolar es ante todo una comunidad de aprendizaje o en aprendizaje, es decir, organizada en equipos de trabajo que permitan administrary mediar la sobrecarga de informacion existente, no solo en el ambito de la ciencia, sino en lo que respecta a toda la informacion que puede generar un val or agregado en el proceso de ensenanza-aprendizaje, El docente se considera un pedagogo investigador que debe propiciar aprendizajes significativos en una verdadera mediacion entre la experiencia de los estudiantes, la informacion existente y la producción colectiva de nueva informacion.

Continuando la revisión de los temas de los congresos, se resalta el XI Congreso Internacional sobre Tecnología y Educacion a Distancia, realizado en Costa Rica en 2002, en el que se refl exiono acerca de como los diferentes "campus virtual es" - los "diseños instruccionales" que al gunas veces se consideran panaceas, no son șino medios para elaborar un "modelo educativo", a traves del cual ejerce su funcion integral la universidad. Esta función no ha sido otra que "buscar la verdad y formar al hombre, mediante la ciencia, para que sirva a la sociedad". Por eso la universidad es busqueda de un horizonte de pensamiento critico y creativo, es formación integral que incluye contenidos y valores, es desarrollo de la ciencia, la "buena ciencia".

En este sentido, la educación superior a distancia es, sobre todo, un "sistema" muy complejo integrado por diversos componentes de cuya interacción y regulación precisas, como en los sistemas biologicos, depende su supervivencia y evolución.

A nalizando los congresos seleccionados en el área de Ética y valores, el tema que sobresale es el de la autonomia. A proposito de ello, el Congreso sobre significado y esencia de la autonomía universitaria en América Latina tuvo, lugar en el auditorio Efrén C. del P,ozo de la Union de Universidades de A merica Latina, Udual, en Ciudad de M exico. En su organizacion participaron en forma conjunta la U dual y la Universidad N acional A utonoma de M exico, Unam. EI Congreso fue inaugurado por el Doctor Guillermo Soberon A cevedo, rector de la Unam, quien exhorto a los asistentes a que realizaran este tipo de investigaciones y que los trabajos del, evento contribuyeran al enriquecimiento y una mejor comprension de la autonomía universitaria en los páises de A merica Latina. 
Revista de

investigaciones UNAD

Volumen 10. Número 1. Junio 2011

En las conclusiones del evento se dice que en el contexto latinoamericano la autonomia es un factor fundamental dentro de la lucha de los pueblos, ya que "ella significa - como apunto el peruano Francisco M iro Quezada - que creemos en a libertad, que consideramos que a libertad de pensamiento, de expresion, de investigacion y de difusion de la cul tura en sus más altas manifestaciones, es una condicion necesaria de la plenitud de la vida social".

El Doctor Leopoldo Zea manifestó que las características de la autonomía de las universidades latinoamericanas coinciden con las de las sociedades en que ella aparece; anadio que la autonomia es una profundizacion del proceso liberador iniciado por los hombres que lucharon por la independencia de estas naciones americanas. Señalo la posibilidad y el deber de la universidad de participar en politica, no entendida en su sentido partidista, de facciones o grupos.

F rente a lo anterior se plantearon diversos interrogantes como estos: ihay al guna relacion entre universidad y politica? ¿L a autonomia reclama el derecho a la critiça y a la accion de los universitarios? ¿Cual es la relacion entre autonomia y accion pol itizadora del pueblo por parte de los estudiantes? Para cerrar, "politizar al pueblo es enseñarlo a razonar, a utillizar la critica frente a cualquier consigna 0 doctrina", enfatizo el Doctor Zea.

2. Resultados de la producción científica por países según las categorías establecidas

Los autores de artículos científicos publicados y analizados en esta investigación provienen de diferentes paises, del mundo, y en razon de su procedencia podemos senalar, los que mayor interés demuestran en el desarrollo de la virtualidad, situacion que se representa en la figura 3.

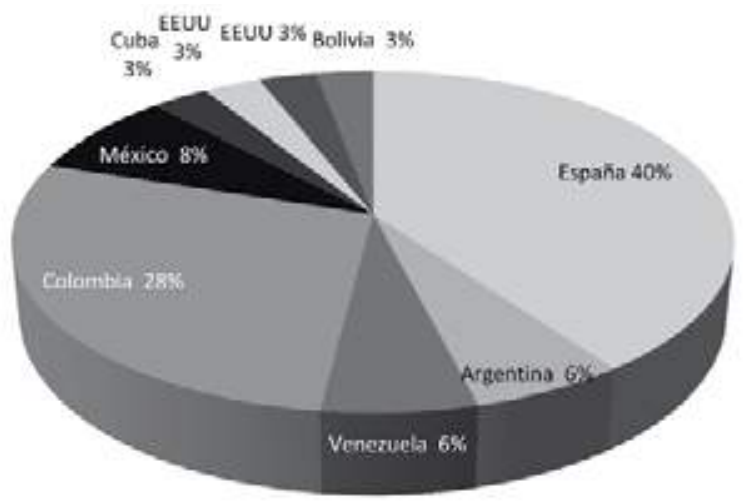

Figura 4. Porcentaje de publicaciones por país

Fuente: Construcción propia de las autoras 


\section{DigualnésPáżGrddbyMaríEEggriaNavesRos}

Aproximación al estado del arte de procesos de aprendizaje en educación virtual, artículo producto de la investigación

España es el país que presenta mayor número de artículos publicados en lo que va de la indagación hasta el momento (40\%). Como caracteristica digna de mencionar, es el unico pals que presenta investigaciones sobre las cuatro categorias (pedagogica, didáctica, calidad y etica y valores). Los demas palses en terminos generales presentan investigaciones en 10 didactico y lo pedagogico.

Las categorías de calidad y ética y valores se han venido socializando hasta lo investigado en el momento, en Espana, A rgentina y Colombia.

M éxico, por su parte, aparece promoviendo la investigación en ambientes virtuales desde lo didáctico; es muy alto el volumen de articul os acerca de los medios y modos de aprendizaje virtual. También se encuentran investigaciones desde la categoria pedagogica. A su vez, Yenezuela está tratando temas tan importantes como la investigacion y gestion del conocimiento en ambientes virtuales, y esta haciendo estudios acerca de la tendencia de estos ambientes en A merica Latina y el Caribe.

Entre los países que más han aportado desde las tesis e investigaciones, se mencionan en su orden: Espana con $25 \%$ de la produccion y con temas especialmente relacionados con la categoria Pedagogía' Colombia, nos enriquece con un $25 \%$ y con temas relacionados igual mente con la categoria Pedagogla.

Luego aparecen Estados Unidos con un $13 \%$ de la producción en esta misma categoria y Venezuela con $13 \%$ en temas relacionados con la didactica, y finalmente Chile con un 12\%, con temas relacionados directamente con pedagogla Figura 5.

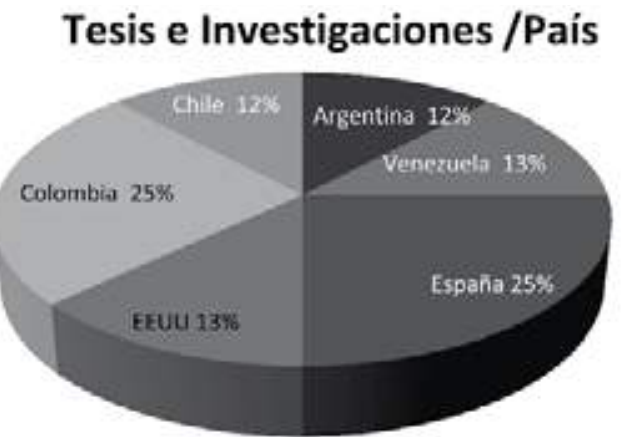

Figura 5. Porcentaje de publicaciones tesis e investigaciones por país Fuente: Construcción propia de las autoras

Los congresos a juzgar por la publicación de sus respectivas memorias y por los paises anfitriones, en cuanto a la reflexion acerca de temas pertinentes a la categoría Pedagogía, fueron liderados por Colombia, Costa Rica y A rgentina, 
Revista de

investigaciones UNAD

Volumen 10. Número 1. Junio 2011

cada uno de ellos con una presencia del 10\%. España exhibió el $60 \%$, dedicado este ultimo ano a la didactica, y por último, México con un $10 \%$, al liderar los congresos que enfatizan en aprendizaje virtual y su relacion con la ética y los valores, resal tando entre ellos la autonomia, Los eventos en A rgentina se centraron en cuatro ejes tematicos: Panoramica internacional, politicas educativas; tecnologla educativa y redes educativas, Figura 6 .

\section{Congreso / País}

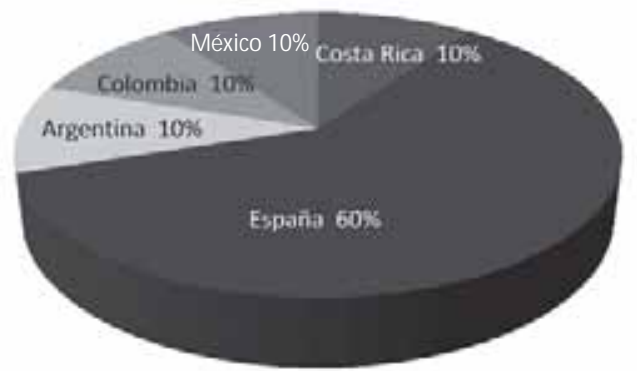

Figura 6. Porcentaje de publicaciones de memorias de congresos por países Fuente: Construcción propia de los autores

Con base en los eventos y memorias consultados hasta la fecha, se puede afirmar que España va a la vanguardia frente a este tipo de programas que tienen como objeto las Tecnologias de la Informacion y la Comunicacion. Los eventos academicos han girado al rededor de las TIC en asuntos como estos: El intercambio de experiencias con sus usos, aplicaciones y posibilidades; su implementacion en universidades, empresas e institutos y las perspectivas innovadoras respecto a la integracion de las TIC en el aula.

Seguidamente se mencionan al gunos de los tantos eventos que se llevaron a cabo:

- II Congreso Internacional Innovamos juntos: Salamanca (España), Mayo 2009.

- Segundas Jornadas de Innovación Docente aplicada a las Tecnologías de la Intormacion y las Comunicaciones: A lcalà (Espana), A bril 2009.

- Primeras Jornadas Ibéricas sobre TIC aplicadas a la Educación, M ovilidad V'irtual y Sociedad del Conocimiento: Granada (España), A bril 2009.

- VIII edición de Expoelearning 2009 con el lema "La imagen del e-learning": B arcelona (España), M arzo 2009. 


\section{DigadilnésPázGrddbyMariaEtgriaNbuesRos}

Aproximación al estado del arte de procesos de aprendizaje en educación virtual, artículo producto de la investigación

- Congreso Internacional sobre uso y buenas prácticas con TIC: Web 2.0: Málaga (España), M arzo 2009.

\section{Conclusiones}

Con fundamento en las diversas fuentes consultadas hasta la fecha, se puede inferir que las areas mas desarrolladas e investigadas en procesos de aprendizaje en educacion virtual son aquellas clasificadas en las categorias Pedagogla y Didactica, que promueven la reflexión desde el saber como fundamentacion y desde el hacer como mejora de las transformaciones que exige este tipo de educacion en el, quehacer discente y docente. Esta primacia se observa tanto en la publicación de artícul os como de tesis, proyectos de investigacion y temas de congresos.

En dicha búsqueda o exploración no se han hallado tesis, proyectos de investigación o congresos cuyos ejes centrales residan en la calidad de la educacion virtual. La reflexion acerca de la cali dad de la educacion virtual se ha promovido fuertemente solo en el terreno de la publicacion de artícul os. Cabe destacar que el gobierno colombiano que desde la normatividad se encuentra en deuda con la educacion a distancia, al mirarla como un enfoque o estrategia de organizacion, ha venido mostrando en los ultimos años un interes y una preocupacion especial, centrada en la calidad de la educacion superior tanto presencial como virtual Ello se ha traducido en avances en cuanto al incremento de la oferta de programas y de la cobertura de la educacion virtual con calidad.

En la categoría Ética y valores en aprendizajes virtuales no aparecen tesis ni proyectos de investigación concluidos pero sí se encontro una serie de articulos y congresos en relacion con la tematica. En diversos paises a promocion de congresos es relativamente reciente y han versado sobre os valores sociales y la etica en la virtualidad, incluyendo temas como la inclusion social.

España y, Colombia, sin duda, son los dos países que encabezan la celebración de congresos, la producción investigativa y las publicaciones que tienen como referentel la educación virtual. Se hace necesario replantear el quehacer pedagogico como base para formar al nuevo ciudadano. El abordaje de temas de la educacion superior virtual se debe promover desde las lineas de investigacion de maestrías, doctorados y de tesis de grado, para que aporten nuevo conocimiento que facilite, fundamente y apoye la toma de decisiones pertinentes en cuanto a normatividad desde las esferas política y curricular. 


\section{Revista de investigaciones UNAD \\ Volumen 10. Número 1. Junio 2011}

\section{Referencias bibliográficas}

Acesad-Icfes-Unisur (1996) Propuestas de políticas para la educación a Distancia, [en línea]. Bogotá. Disponible en: acesad.org.co.pdf.presenta-05.pdf. [2010, 7 de abril].

Anzola, M., O. L. (2000). ¿Por qué es importante indagar lo pedagógico en el Sistema de Educación Abierta y a Distancia? Bogotá: UNAD.

Arias, J. et al. (1983). Universidad a Distancia: del sueño a la realidad. Bogotá: Fundación para la Educación Superior.

Burgos, J. (2004). Hacia un modelo de quinta generación en educación a distancia. Una visión de competencia con perspectiva global, [en línea]. Ciudad de México. Disponible en: http://www.ateneonline.net/datos/15_03_Burgos_Vladimir.pdf. [2010, 7 de mayo].

Cardona, G. (2008). Tendencias educativas para el siglo XXI: Educación virtual, online y e learning. Revista Electrónica Educativa de Tecnología, Septiembre.

Facundo, Á. (2004). La virtualización desde la perspectiva de la modernización de la educación superior: Consideraciones pedagógicas. Revista de Universidad y Sociedad del Conocimiento, RUSC.

Gómez, G. \& Martí, D. (2005). M étodo de una investigación en comunicación: Fuentes primarias y card sorting para identificar y agrupar en niveles los criterios de evaluación más utilizados en festivales web. Revista Textos de la cibersociedad, 7.

González, A. (2005). Las tecnologías de la información y la educación, [en línea]. Observatorio para la cibersociedad. Disponible en: http://www.cibersociedad.net/ archivo/articulo. php?art=201[2006, 7 de mayo].

González, B., E. Formación del Tutor para la Educación a Distancia y los ambientes virtuales de aprendizaje en la Universidad de Colombia 1974-2002. Bogotá: Rudecolombia.

Landazábal, D. (2005). Mediación en entornos virtuales de aprendizajes, análisis de las estrategias metacognoscitivas y de las herramientas comunicacionales. Bogotá: Universidad del Bosque.

Lorenzo, G., A. (1999, junio). Historia de la Educación a Distancia. Revista Iberoamericana de Educación a Distancia, RIED, 2(1).

Rodríguez, C. (2005). Hipertexto y literatura en Red. El relato digital, los escrilectores y la Cibercultura, Revista Textos de la cibersociedad [en línea], 7. Disponible en: http:// www . cibersociedad. net/textos/articulo.php?art=81 [2009, 7 de junio]. 


\section{DigualnéśáżGrddbylMaíaEtgariaNbuesRos}

Aproximación al estado del arte de procesos de aprendizaje en educación virtual, artículo producto de la investigación

Santillán, C., F. (2006). El Aprendizaje basado en problemas como propuesta educativa de las disciplinas económicas y sociales apoyadas en el B learning. Revista Iberoamericana de Educación [en línea]. Disponible en: http://www.rieoei.org/delos lectores/1460Santillan. pdf [2009, 10 de octubre].

Silva, J . (2006). Formación docente en un espacio virtual de aprendizaje: Una experiencia concreta en el contexto chileno. En López Ricardo (Coord.) Educación matemática y tecnologías de la información [monográfico en línea]. Revista electrónica Teoría de la Educación y Cultura en la Sociedad de la Información. 7(1), 1006.

Valdés, M., C., De Armas, N. Darin, S., B., Abreu, M . y Castro, A. (2008). Una herramienta TIC estratégica para el crecimiento profesional en la sociedad del conocimiento: La formación transversal curricular de competencias comunicativas. EDUTEC. Revista Electrónica de Tecnología Educativa [en línea], 26. Disponible en: http://cent.uji.es/ octeto/node/2130. [2010, 7 de marzo].

Congreso Internacional sobre uso y buenas prácticas con TIC: Web 2.0. Málaga, España. Marzo de 2009. http://congresotic.uma.es/

I Congreso virtual Latinoamericano de Educación a Distancia. Argentina. Marzo 23-abril 4 de 2004. http://www.latineduca.com/latineduca/arg/Congreso Internacional El profesorado ante el reto de las nuevas tecnologías en la Sociedad del Conocimiento. Granada, España. Marzo de 2005. http://www.edutec.es/informes/memoriaees.pdf

II Congreso Internacional Innovamos juntos. Salamanca, España. Mayo de 2009. http://www.csi-csif.es/innovamosjuntos/.

III Congreso ONLINE OCS. www.cibersociedad.net/congres2006.

Expoelearning 2009 La imagen del e-learning. Barcelona, España. Marzo de 2009. http://www.expoelearning.com.

Primeras Jornadas Ibéricas sobre TIC aplicadas a la Educación, Movilidad Virtual y Sociedad del Conocimiento Granada, España. Abril de 2009. http://www.recbib. es/event/i-jo rnadas-ibericas-sobre-tic-aplicadas-a-la-educacion-movilidad-virtual-ysociedad-del-conocimiento.

Segundas Jornadas de Innovación Docente aplicadas a las Tecnologías de la Información y las Comunicaciones. Alcalá, España. Abril de 2009. http://ssp.uah.es/ idtic/jornadas/ programa.html 